Documentation et bibliothèques

DOCUMENTATION BIBLIOTHËQUES

\title{
Le réaménagement du Secteur droit et science politique de la Bibliothèque de l'Université Laval
}

\section{Christine Lachance}

Volume 46, numéro 3, juillet-septembre 2000

URI : https://id.erudit.org/iderudit/1032656ar

DOI : https://doi.org/10.7202/1032656ar

Aller au sommaire du numéro

Éditeur(s)

Association pour l'avancement des sciences et des techniques de la

documentation (ASTED)

ISSN

0315-2340 (imprimé)

2291-8949 (numérique)

Découvrir la revue

Citer cet article

Lachance, C. (2000). Le réaménagement du Secteur droit et science politique de la Bibliothèque de l'Université Laval. Documentation et bibliothèques, 46(3),

145-148. https://doi.org/10.7202/1032656ar

Tous droits réservés (c) Association pour l'avancement des sciences et des techniques de la documentation (ASTED), 2000
Ce document est protégé par la loi sur le droit d'auteur. L'utilisation des services d'Érudit (y compris la reproduction) est assujettie à sa politique d'utilisation que vous pouvez consulter en ligne.

https://apropos.erudit.org/fr/usagers/politique-dutilisation/ 


\title{
Le réaménagement du Secteur droit et science politique de la Bibliothèque de l'Université Laval
}

\author{
Christine Lachance \\ Conseillère à la documentation en Bioéthique, droit et science politique \\ Coordonnatrice de la collection en Bioéthique et Études internationales \\ Droit et science politique \\ Bibliothèque de l'Université Laval
}

Cette chronique présente un aperçu du réaménagement des collections et des services documentaires en droit et science politique qui conduisit à la création du Secteur droit et science politique en 1996.

Ce réaménagement est issu de la concertation entre la Faculté de droit, le Département de science politique et la Direction de la Bibliothèque pour remédier au manque d'espace, à l'accroissement et la diversification de la collection ainsi que pour faire face à l'émergence des nouvelles technologies.

La bibliothèque du Secteur droit et science politique a comme mission d'assurer le soutien documentaire pour les études en droit et science politique. Elle doit mettre à la disposition des professeurs, des chercheurs et des étudiants inscrits aux trois cycles d'études au sein des deux facultés, une collection documentaire et les services professionnels requis pour les fins d'enseignement et de recherche. Ceci exige une étroite collaboration entre les professeurs et les comités de bibliothèque.

\section{Les locaux}

Le relogement de la collection de droit en 1995 a permis de réaménager les diverses composantes de la collection et de créer un espace spécialisé et agréable pour le Secteur droit et science politique à l'intérieur de la Bibliothèque de l'Université Laval située au centre du campus, pavillon Jean-Charles-Bonenfant. L'ensemble des collections, des services aux usagers et le personnel de la bibliothèque $y$ sont regroupés.

La clientèle a ainsi accès en un même lieu à toute la documentation disponible, que ce soit les ouvrages de droit, les documents parlementaires (journaux officiels, débats, etc.), les publications des NationsUnies et les traités de science politique.
Le Secteur droit et science politique occupe le $2^{e}$ étage de la Bibliothèque de l'Université Laval. Dès son arrivée, le visiteur a accès sur sa gauche à un espace regroupant les locaux où se retrouvent plusieurs services:

a un laboratoire d'informatique financé entièrement par le fonds d'investissement étudiant de droit et le bureau du responsable du laboratoire ${ }^{1}$;

- une réserve où les professeurs peuvent déposer les ouvrages essentiels pour les cours offerts durant le trimestre;

- une collection de référence de base, ouvrages juridiques récents, grands traités politiques, manuels, projets de lois, Gazette officielle, dictionnaires, encyclopédies, livraisons courantes des principaux périodiques juridiques canadiens et recueils de jurisprudence.

On y trouve aussi une vaste salle de classe pouvant accueillir une cinquantaine d'étudiants où se donne la formation méthodologique et regroupant un exemplaire des principaux index, bibliographies, etc. Les professeurs viennent $y$ enseigner les fondements méthodologiques de la recherche et de la rédaction à proximité des collections.

Dans l'espace attenant à la salle de classe se retrouvent les micro-ordinateurs à partir desquels les étudiants peuvent consulter différentes banques de données, le catalogue Ariane, les pages de ressources ainsi que des tables de travail où les étudiants ont la possibilité de travailler en équipe. On y expose aussi chaque semaine les nouveautés.

Le reste de l'étage est occupé en grande partie par les livres et les périodiques et l'on a pris soin d'intercaler, entre des groupes de rayons, des isoloirs. Les collections sont organisées selon la classification de la bibliothèque du Congrès; viennent en premier périodiques, bibliographies, ouvrages de droit comparé, puis ceux de droits nationaux, documents parlementaires et ouvrages de doctrine.

Les cabinets de travail réservés aux étudiants de doctorat et de maîtrise sont répartis tout autour de l'étage. On a aussi aménagé une salle de travail pour les étudiants de $2^{e}$ cycle ainsi qu'une salle pour les professeurs qui désirent travailler en toute quiétude. Des locaux pour le travail en groupe sont aussi disponibles.

\section{Les collections}

Les collections contiennent des ouvrages juridiques et politiques qui reflètent bien la situation politique et juridique du Québec. Elles se veulent exhaustives en droit public canadien, en droit fédéral et en droit québécois.

On trouve également des collections de base et de recherche (législation, jurisprudence, doctrine) en droit anglais et français en raison du rôle supplétif de ces droits dans le système judiciaire canadien, et une collection de base et de recherche en droit américain et européen (Union européenne). Ce qui permet des études de $2^{\mathrm{e}}$ cycle et même de $3^{\mathrm{e}}$ cycle en droit comparé pour ces pays et régimes.

\section{Points forts des collections}

Première université francophone d'Amérique, l'Université Laval se doit de conserver et développer une collection documentaire en langue française. Aussi, y achète-t-on prioritairement, quels que soient le sujet et la juridiction, des ouvrages en langue française. L'histoire nous a légué un régime civiliste et nous possédons une prestigieuse collection d'ouvrages classiques de droit civil français (XVIIe

1. Un laboratoire d'informatique pour les étudiants de science politique est en voie de réalisation grâce à la contribution du fonds d'investissement des étudiants en science politique. 
et XVIIIe siècle). Cette collection de droit civil est sans cesse actualisée.

Au cours des vingt dernières années, la pratique du droit s'est considérablement diversifiée et l'aire d'activité des juristes s'est étendue graduellement du Canada au continent nord-américain, puis au monde entier. Des secteurs nouveaux ont pris de plus en plus d'importance en raison des préoccupations sociales (environnement, bioéthique, questions autochtones), des accords commerciaux ${ }^{2}$, (droit du travail comparé) et des événements politiques (droit pénal international).

Les domaines correspondant aux secteurs d'excellence dans l'enseignement et la recherche à l'Université Laval constituent nos priorités de développement. Ces secteurs sont l'environnement au sens large: agriculture, aménagement du territoire, ressources naturelles, protection de l'environnement, droit pénal de l'environnement, etc., le droit commercial international, le droit du travail international et comparé, le droit pénal international et comparé, les questions autochtones et la bioéthique.

\section{Les collection par thèmes}

\section{Publications officielles}

Dans un premier temps, pour répondre aux besoins documentaires diversifiés de la clientèle, nous acquérons les documents officiels de différents organismes et gouvernements.

La Bibliothèque est dépositaire des publications en langue française des $\mathrm{Na}$ tions Unies ${ }^{3}$. Elle achète toutes les publications de l'Union européenne et de l'Organisation internationale du travail et une sélection des documents du Conseil européen, de l'Union interparlementaire, de I'Union internationale de conservation de la nature, de l'Organisation mondiale de la santé (OMS), du Programme des Nations Unies pour l'environnement (PNUE), de l'Organisation des Nations Unies pour l'alimentation et l'agriculture (FAO) ainsi que de l'Organisation de coopération et de développement économique (OCDE).

Dans le passé, l'achat de documentation était limité géographiquement à celle de la Grande-Bretagne, des États-Unis et de la France; cependant, depuis quelques années, la documentation des pays d'Amérique latine fait aussi partie de nos acquisitions. Par conséquent, les volumes en fran- çais et en anglais se retrouvent maintenant avec des ouvrages en langue espagnole.

\section{Environnement}

La collection inclut des ouvrages sur la protection de l'environnement au niveau international ainsi que d'autres décrivant les politiques nationales. Une attention toute particulière est portée au droit et à la politique de l'eau, de l'énergie et des ressources naturelles. Des recueils de traités internationaux et de textes normatifs (soft law, codes de conduite, etc.) sont également acquis. La collection comporte de plus des ouvrages spécialisés concernant le droit et les politiques applicables aux différents thèmes: eau, énergie, ressources naturelles, gestion des déchets, développement durable, aménagement du territoire, éthique de l'environnement, mouvements écologistes.

\section{Droit commercial international}

La Bibliothèque possède des recueils de formulaires internationaux 4 en français et en anglais qui permettent aux étudiants d'examiner toutes les formes de contrats (franchise, concession, savoir-faire ou knowhow, coentreprise ou joint venture, transfert de technologie), etc. On retrouve également des ouvrages sur les contrats internationaux, le droit du commerce international ainsi que des ouvrages spécialisés sur le droit des franchises, cession de marque et valeurs mobilières, etc., dans différents pays ${ }^{5}$. Et nous achetons les recueils sur les accords commerciaux et leurs applications.

\section{Autochtones}

En droit des autochtones, la collection offre des ressources documentaires pour l'étude non seulement du cadre juridique et politique s'appliquant aux autochtones d'Amérique du Nord, mais aussi à ceux d'Amérique latine et d'Europe.

\section{Droit bancaire comparé et international}

D'autres domaines sont en expansion, soit le droit bancaire comparé international, le droit comparé et international du travail et le droit pénal comparé et international.

\section{Régimes politiques des pays d'Amérique latine}

Dans un souci d'adaptation du Canada à des ensembles économiques et à des politiques continentales, le développement d'une collection sur les systèmes juridiques et politiques des pays d'Amérique latine (accords commerciaux reliés au Mercosur, Marché commun du sud [de l'Amérique], autochtones, droit constitutionnel, régimes politiques, protection de l'environnement, etc.) est en cours.

\section{Le personnel}

Pour permettre à la clientèle d'avoir accès rapidement et efficacement à l'information, quelques personnes animent le secteur. Le personnel comprend trois juristes de formation, soit un juriste rattaché à la Faculté de droit et responsable du laboratoire d'informatique ainsi que deux bibliothécaires 6 qui se répartissent les tâches d'aide à l'enseignement et à la recherche, le développement des collections, la communication de l'information et la formation documentaire. Ces personnes sont assistées par une équipe de commis qui assurent en permanence le service à la réserve et le replacement des volumes.

Les deux bibliothécaires se partagent les tâches et les disciplines. L'un s'occupe principalement du droit. II est responsable de l'édition de la page Web en droit et du bulletin AJOUR. L'autre personne travaille

2. Organisation mondiale du commerce (OMC), Accord de libre-échange nord-américain (ALÉNA).

3. Publications en langue française déposées au Secrétariat de l'Assemblée générale.

4. Fédération internationale des ingénieurs-conseil (FIDIC), Chambre de commerce international (CCI), Commission des Nations Unies pour le droit commercial international (CNUDCI).

5. Dictionnaire foly: pratique des contrats internationaux. Paris: GLN/Joly éditions.

$L A M Y$ contrats internationaux. Paris: Lamy.

Law and practice under the GATT. New York: Oceana publications.

Law \& practice of the World Trade Organisation. New York: Oceana publications.

International contract manual: country bandbooks. Boston, Deventer: Kluwer Law and Taxation Publishers. International agency and distribution law. New York: M. Bender.

Digest of commercial laws of the world. Dobbs Ferry, New York: National Association of Credit Management.

International contract manual: contract checklist. Deventer, Boston: Kluwer Law and Taxation Publisher.

6. Le titre de la fonction est conseiller à la documentation. 
plus spécialement en droit de l'environnement, des autochtones, du droit bancaire, des sciences et du droit pénal ; elle est responsable de la science politique et des relations internationales et conséquemment, de l'édition des pages Web en science politique, études internationales et bioéthique.

\section{Les outils documentaires}

Ces conseillers produisent toute une gamme d'outils bibliographiques visant à faciliter l'accès à l'information pour la clientèle. Tous ces outils sont disponibles dans Internet dans le cadre de pages de ressources spécialisées en droit, en politique, en bioéthique et en relations internationales. Ce sont les biblioguides (bibliographies spécialisées mises à jour régulièrement), Bidduls ${ }^{7}$ et Bipo/s 8 , deux bulletins d'information thématique et ponctuelle sur des sujets d'actualité en droit et en science politique. Les biblioguides couvrent un secteur du droit ou de la science politique (par exemple, l'agriculture, le droit médical, les systèmes politiques comparés) et présentent l'ensemble des sources documentaires disponibles à la Bibliothèque (dictionnaires, encyclopédies, recueils de jurisprudence, recueils de lois, traités, ouvrages de vulgarisation, etc.). Les Bidduls et Bipols présentent la documentation concernant un sujet d'actualité. Leur format est normalisé (une page) et ils ne sont pas mis à jour, contrairement aux biblioguides. De plus, les conseillers offrent des services d'information par courrier électronique, soit le bulletin AJOUR (Actualité juridique organisée en unité de référence) et les courriels: Courrie/ $\mathrm{BIO}^{9}$ et Courriel D.R.O.I.T. Le bulletin AJOUR consiste en une notule d'information sur un sujet d'actualité juridique canadienne, très récent. Les Courriels permettent de diffuser l'information quels que soient le support documentaire, la nature et la provenance dans les domaines éthiques, juridiques et politiques couverts par la recherche et l'enseignement à l'Université Laval.

Courriel D.R.O.I.T. traite des principaux secteurs du droit et Courriel BIO couvre les sujets pluridisciplinaires (autochtones, environnement, bioéthique, santé, protection sociale, agriculture, sciences) des points de vue juridique, poli- tique et éthique. II s'agit de deux services d'information électronique sur les principaux développements affectant ces domaines (projets de loi, commissions d'enquête, sites Web intéressants, ouvrages d'intérêt, etc.); ils sont offerts gratuitement aux membres de la communauté universitaire.

Le service LIVRES sert à informer mensuellement les professeurs des nouvelles acquisitions tant en droit qu'en science politique.

Outre leurs fonctions en développement des collections et en communication de l'information, les conseillers à la documentation contribuent à la formation documentaire par leur participation aux cours de méthodologie de la recherche 10 aux 1 er et $2^{e}$ cycle ou par des séminaires de formation documentaire donnés tant dans le cadre des cours spécialisés à la demande des professeurs que par un programme annuel de séminaires ${ }^{11}$. Ces séminaires, d'une durée de 3 heures visent à présenter aux étudiants et aux chercheurs, les principales ressources documentaires sur support traditionnel et électronique.

Les séminaires de formation documentaire sont des exposés oraux de trois heures comprenant une présentation de la documentation, qui se trouve sur place et sur écran, du catalogue de la bibliothèque, des sites Internet, des banques de données disciplinaires et bibliographiques. Ces séminaires permettent de présenter, pour un domaine précis du droit ou de la science politique, l'ensemble des ressources documentaires disponibles à la Bibliothèque ou dans Internet ainsi que les principaux acteurs (organisations non gouvernementales, organismes législatifs) du domaine.

Bref, les conseillers travaillent en étroite collaboration avec le corps professoral et les Comités de bibliothèque ${ }^{12}$. Ceux-ci sont très présents dans la gestion du Secteur droit et science politique. Les questions importantes telles que budget, politique de développement, etc., sont portées à leur attention et les Comités sont très sensibles aux problèmes budgétaires de la Bibliothèque. Les conseillers en sont membres d'office et ils assurent la liaison entre la Faculté de droit et la Bibliothèque de l'Université Laval, et entre le Département de science politique, d'une part, et la Bibliothèque d'autre part. Les conseillers assurent l'aide et les supports documentaires à l'enseignement et à la recherche. Ils contribuent à la formation des juristes et politiciens de demain.

\section{Le développement des collections : une préoccupation constante}

Fière de ses origines historiques et de son caractère francophone solidement ancrée dans le continent nord-américain, mais ouverte sur le monde à l'image de l'Université Laval, la Bibliothèque, tout particulièrement le Secteur droit et science politique, envisage l'avenir avec sérénité. Tout en conservant ses richesses patrimoniales (anciens documents parlementaires britanniques, ouvrages anciens de droit français) et sa collection de livres, elle sait utiliser les nouvelles technologies de l'information. Désormais toutes les disciplines s'appuient sur les ressources Internet et tous les outils documentaires sont disponibles par l'intermédiaire des serveurs Web. Notre collection précède les développements politiques et juridiques de l'actualité pour permettre aux finissants d'être prêts pour affronter les problèmes de demain.

Cependant, le développement des collections est une préoccupation constante considérant les compressions budgétaires des dernières années qui nous ont forcé à annuler plusieurs abonnements. Jusqu'à maintenant, il a été possible de

7. Bidduls: Bulletin d'information sur la documentation en droit de l'Université Laval.

8. Bipols: Bulletin d'information sur la documentation en science politique de l'Université Laval.

9. Courriel BIO est produit conjointement par la conseillère en droit et bioéthique et le conseiller en psychologie et bioéthique.

10. Travail dirigé en science politique et Méthodologie de la recherche juridique.

11. No 89-1999, Nations Unies, 23 septembre 1999, Denis Le May.

No 90-1999, Ressources naturelles, énergie, faune, flore, droit international, 14 octobre 1999, Christine Lachance.

No 91-1999, Droit pénal comparé et international, 18 novembre 1999, Christine Lachance et Denis Le May.

$\mathrm{N}^{\circ}$ 92-2000, Droit autochtone, 20 janvier 2000, Christine Lachance.

$\mathrm{N}^{\circ}$ 93-2000, Droit de la culture et du divertissement, 17 février 2000, Denis LeMay.

No 94-2000, Droit international, 16 mars 2000, Denis LeMay.

12. Droit et Science politique. 
préserver les secteurs de pointe en diminuant les acquisitions d'ouvrages généraux ou dans les secteurs secondaires. Les contributions facultaires (droit et science politique) nous ont permis de maintenir les secteurs de pointe. Les sommes versées par le fonds des étudiants de droit a assuré le renouvellement des collections d'enseignement de 1 er cycle, mais de nouveaux investissements sont requis pour maintenir le niveau des collections et pour pouvoir acheter la documentation nécessaire à l'enseignement des nouveaux programmes et pour la recherche.

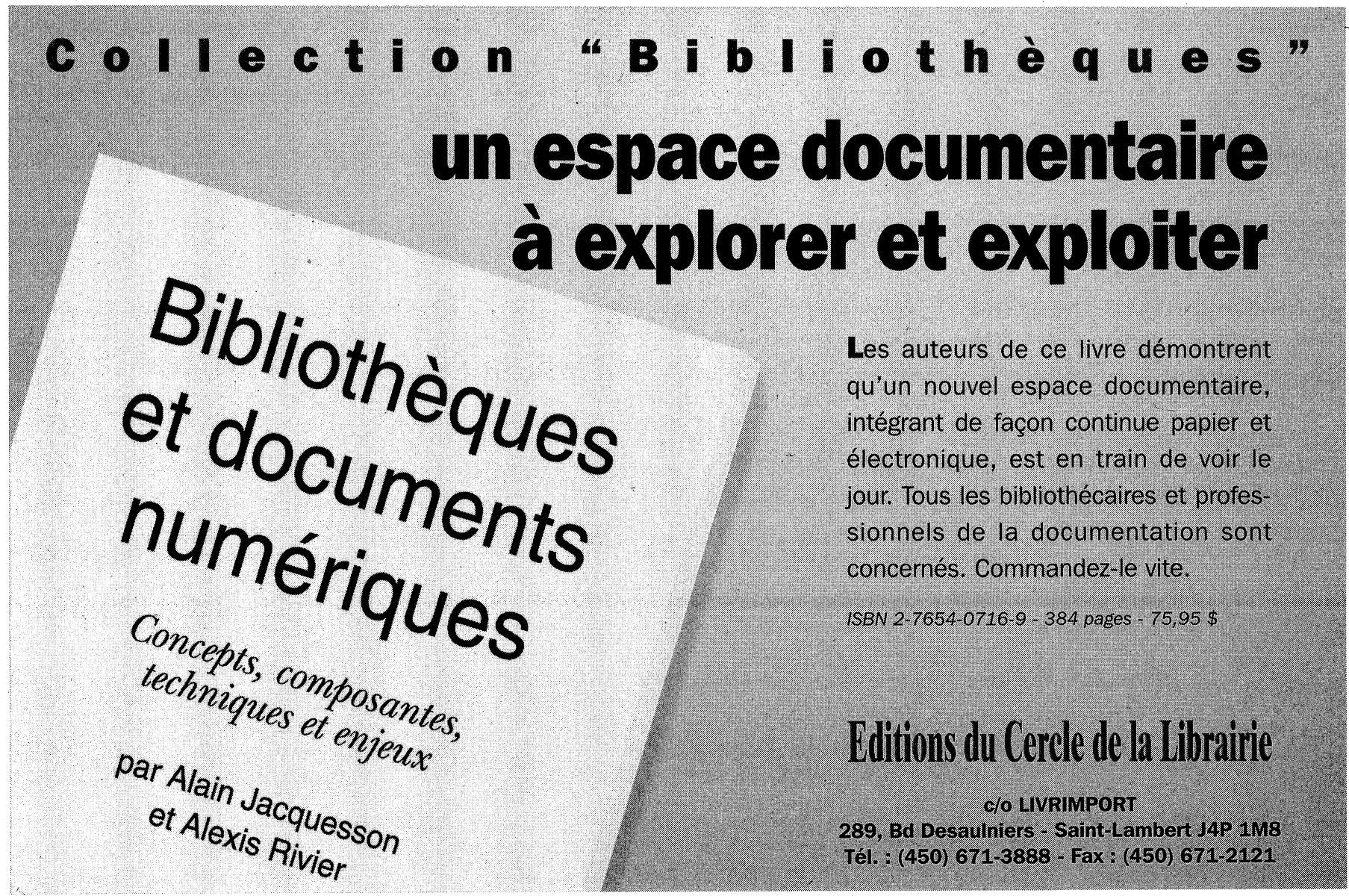

